Received: 30.11 .2020

Revised: 01.02.2021

Accepted: 26.02 .2021

DOI: $10.17804 / 2410-9908.2021 .1 .055-063$

\title{
ON ONE PROBLEM OF DETERMINING THE OPTIMAL RESIDUAL STRESS FIELD
}

\author{
V. V. Struzhanov \\ Institute of Engineering Science, Ural Branch of the Russian Academy of Sciences, \\ 34, Komsomolskaya St., 620049, Ekaterinburg, Russian Federation \\ iD http://orcid.org/0000-0002-3669-2032 ख stru@imach.uran.ru \\ *Corresponding author: E-mail: stru@imach.uran.ru \\ Address for correspondence: 34 Komsomolskaya St., 620049, Ekaterinburg, Russian Federation \\ Tel.: +7(343)362 3019
}

An operator equation is obtained, the solution of which is an intrinsic (residual) stress tensor reducing the stress level to zero in a predetermined region of a rigidly loaded elastic body. It is shown that the operator of this equation is a contraction operator and, therefore, this equation can be solved by the method of successive approximations. An example is given.

Keywords: residual stresses, energy Hilbert space, orthogonal subspaces, operator equation, contraction operator, successive approximations.

\section{References}

1. Birger I.A. Ostatochnye napryazheniya [Residual Stresses]. Moscow, Mashgiz Publ., 1963, 262 p. (In Russian).

2. Pozdeev A.A., Nyashin Yu.I., Trusov P.V. Ostatochnye napryazheniya: teoriya $i$ prilozheniya [Residual Stresses: Theory and Applications]. Moscow, Nauka Publ., 1982, 111 p. (In Russian).

3. Pavlov V.F., Kirpichev V.A., and Ivanov V.B. Ostatochnye napryazheniya i soprotivlenie ustalosti uprochnennykh detalei s kontsentratorami napryazhenii [Residual Stresses and Fatigue Resistance of the Reinforced Components with Stresses Concentration]. Samara, SNTs RAN Publ., 2008. (In Russian).

4. Abramov V.V. Ostatochnye napryazheniya i deformatsii v metallakh [Residual Stresses and Strains in Metals]. Moscow, Mashgiz Publ., 1963, 355 p. (In Russian).

5. Korolev A.V., Mazina A.A., Yakovishin A.S., Shalunov A.V. Technological causes of residual stresses. Sovremennye Materialy, Tehnika i Tekhnologii, 2016, vol. 5 (8), pp. 116-119. (In Russian).

6. Struzhanov V.V. Determination of shrinkage stresses in components of stochastically reinforced composites. Soviet Applied Mechanics, 1982, vol. 18, no. 5, pp. 445-449. DOI: $10.1007 / \mathrm{bf} 00883786$.

7. Shinkin V.N. Residual stresses during expansion of steel pipes. Young Scientist, 2015, No. 20, pp. 88-93. ISSN 072-0297. (In Russian).

8. Kolmogorov G.L., Kuznecova E.V., Tiunov V.V. Tekhnologicheskie ostatochnye napryazheniya i ikh vliyanie na dolgovechnost i nadezhnost metalloizdeliy [Technological Residual Stresses and their Effect on the Longevity and Reliability of Hardware]. Perm, Izd-vo Permskogo nats. issled. politekhnicheskogo un-ta Publ., 2012, 226 p. (In Russian). 
9. Burkin S.P., Shimov G.V., Andryukova E.A. Ostatochnye napryazheniya v metalloproduktsii [Residual stresses in metal products: tutorial]. Ekaterinburg, Izdatelstvo Uralskogo gosudarstvennogo universiteta Publ., 2015, 248 p. (In Russian).

10. Hosford W.F. Mechanical Behavior of Materials. Cambridge, Cambridge University Press, 2005, $421 \mathrm{p}$.

11. Kozic D., Gдnser H.-P., Brunner R., Kienerb D., Antretterc T., Kolednik O. Grack arrest in thin metallic film stacks due to material and residual stress inhomogeneities. Thin Solid Films, 2018, vol. 668, pp. 14-22. DOI: 10.1016/j.tsf.2018.10.014.

12. Konovalov D.A., Vichuzhanin D.I., Smirnov S.V. Evaluating the residual stresses by the method of indentation. In: Proceedings of the Second All-Russian Scientific Conference "Matem. Mod. Kraev. Zadachi”, 1-3 June 2005, part 1, Samara State Technical Univ., Samara, 2005, pp. 155-157. (In Russian).

13. Fishkin A.I. Application of the Tikhonov regularization for residual stresses determination. Stroitelnaya Mekhanika Inzhenernykh Konstruktsij i Sooruzhenij, 2009, No. 4, pp. 48-55.

14. Kudryavtsev I.V. Vnutrennie napryazheniya kak rezerv prochnosti v mashinostroenii [Internal Stresses as a Strength Reserve in Mechanical Engineering]. Moscow, Mashgiz Publ., 1951, 278 p. (In Russian).

15. Rozhkov I.I., Mylnikov V.V. Calculation of internal residual stresses arising in hardened parts of machines after chemical-thermal processing. International Journal of Experimental Education, 2014. vol. 1, pp. 114-118. (In Russian).

16. Sinchurin D.V. The effect of hydraulic shot peening on the increase of the service reliability of parts. Young Scientist, 2015, vol. 21.2 (101.2), pp. 54-57. (In Russian).

17. Lurie A. I. Teoriya uprugosti [Theory of Elasticity]. Moscow, Nauka Publ., 1970, 940 p. (In Russian).

18. Sokolov A.G., Struzhanov V.V. A problem of optimizing the stressed state in an elastic solid. Journal of Applied Mathematics and Mechanics, 2001, vol. 65, iss. 2, pp. 311-316. DOI: 10.1016/S0021-8928(01)00035-1.

19. Mikhlin S.G. Variatsionnye metody v matematicheskoy fizike [Variational Methods in Mathematical Physics]. Moscow, Nauka Publ., 1970, 512 p. (In Russian).

20. Struzhanov V. V. On the method of orthogonal projections in the theory of elasticity. Vestn. Samar. Gos. Tekhn. Univ., Ser. Fiz.-Mat. Nauki [J. Samara State Tech. Univ., Ser. Phys. Math. Sci.], 2017, vol. 21, no. 2, pp. 308-325. DOI: 10.14498/vsgtu1542. (In Russian).

21. Kantorovich A.V., Akilov G.P. Funrtsionalnyi Analiz [Functional Analysis]. Moscow, Nauka Publ., 1977, 744 p. (In Russian).

22. Konstantinov R.V. Lektsii po funktsionalnomu analizu [Lectures on Functional Analysis]. Moscow, MFTI Publ., 2009, 374 p. (In Russian). 
Подана в журнал: 30.11 .2020

УДК 539.3

DOI: $10.17804 / 2410-9908.2021 .1 .055-063$

\title{
ОБ ОДНОЙ ЗАДАЧЕ ОПРЕДЕЛЕНИЯ ОПТИМАЛЬНОГО ПОЛЯ ОСТАТОЧНЫХ НАПРЯЖЕНИЙ
}

\author{
В. В. Стружанов \\ Федеральное государственное бюджетное учреждение науки \\ Институт машиноведения Уральского отделения Российской академии наук, \\ 34, ул. Комсомольская, 620049, г. Екатеринбург, Российская Федерация \\ iDhttp://orcid.org/0000-0002-3669-2032 ख stru@imach.uran.ru \\ *Ответственный автор. Электронная почта:stru@imach.uran.ru \\ Адрес для переписки: ул. Комсомольская, 34, Екатеринбург, Российская Федерация \\ Тел.:+7(343)362-30-19
}

Получено операторное уравнение, решением которого является тензор собственных (остаточных) напряжений, снижающий до нуля уровень напряжений в наперед заданной области упругого тела при его жестком нагружении. Показано, что оператор этого уравнения является оператором сжатия и, следовательно, решение этого уравнения можно найти методом последовательных приближений.

Ключевые слова: остаточные напряжения, энергетическое гильбертово пространство, ортогональные подпространства, операторное уравнение, оператор сжатия, последовательные приближения.

\section{1. Введение}

Остаточными (собственными) напряжениями принято называть такие напряжения, которые существуют и уравновешиваются в теле после устранения воздействий, вызвавших их появление. Уровень остаточных напряжений, возникающих почти при всех технологических процессах термической и механической обработки, является важным параметром, определяющим качество элементов конструкций [1-11]. Эти напряжения, складываясь с напряжениями от внешней нагрузки, либо увеличивают несущую способность конструкционных элементов, либо уменьшают ее $[3,8,12]$. Остаточные напряжения определяют либо экспериментально [12], либо используя современные математические методы [13]. Причем в технике хорошо известны технологии создания благоприятных полей остаточных напряжений $[1,14-16]$. Очевидно, что дать оценку влияния остаточных напряжений на прочность изделий желательно получить уже на стадии проектирования для того, чтобы разработать такую технологию изготовления деталей конструкций, при которой возникали бы остаточные напряжения, благоприятно взаимодействующие с напряжениями, появляющимися в процессе их эксплуатации.

В некоторых случаях необходимо снизить уровень напряжений только в отдельных областях - областях концентрации напряжений от внешней нагрузки. В работе приведена методика расчета такого поля собственных напряжений, которое при заданном жестком нагружении упругого тела, складываясь с напряжениями от нагрузки, позволяет в отдельных областях тела получить суммарное напряженное состояние, сколь угодно близкое к нулю. При этом неявно предполагается, что в других областях суммарные напряжения не превосходят предела текучести. 


\section{2. Постановка задачи}

Рассмотрим упругое тело $V$, ограниченное достаточно гладкой поверхностью Г. Зададим точкам части границы $\Gamma_{1}$ перемещения, определяемые вектором $\boldsymbol{v}(y), y \in \Gamma_{1}$. Оставшаяся часть границы $\Gamma_{2}$ свободна от напряжений. Напряженно-деформированное состояние в теле находится из решения краевой задачи

$$
\nabla \cdot \boldsymbol{\sigma}=0, \boldsymbol{\varepsilon}=\operatorname{def} \boldsymbol{u}, \boldsymbol{\sigma}=\boldsymbol{C} \cdot \boldsymbol{\varepsilon},\left.\boldsymbol{u}\right|_{\Gamma_{1}}=\boldsymbol{v},\left.\boldsymbol{\sigma} \cdot \boldsymbol{n}\right|_{\Gamma_{2}}=0 .
$$

Здесь первая группа уравнений - это уравнения равновесия; вторая - соотношения Коши; третья - закон Гука [17]. Симметричные тензоры второго ранга напряжений и деформаций обозначены, соответственно, символами $\boldsymbol{\sigma}$ и $\boldsymbol{\varepsilon}, \boldsymbol{u}-$ вектор перемещений внутренних точек тела $V ; \boldsymbol{n}$ - единичный вектор внешней нормали к поверхности $\Gamma$; $\boldsymbol{C}$ - симметричный тензор четвертого ранга модулей упругости; $\boldsymbol{\nabla}$ - оператор Гамильтона, точкой обозначено скалярное произведение, двумя точками - двойное скалярное произведение тензоров [10].

Если теперь в теле возникнет поле первоначальных несовместных деформаций [11], заданное симметричным несовместным тензором второго ранга $\varepsilon^{*}(x),[\in V$, то появятся дополнительные самоуравновешенные напряжения [11]. Для их определения необходимо решить краевую задачу [18]:

$$
\nabla \cdot \mathbf{q}^{\prime \prime}=0, \mathbf{e}=\operatorname{def} \boldsymbol{u}, \boldsymbol{q}^{\prime \prime}=\boldsymbol{C} \cdot \cdot\left(\boldsymbol{e}-\boldsymbol{\varepsilon}^{*}\right),\left.\boldsymbol{u}\right|_{\Gamma_{1}}=0,\left.\boldsymbol{q}^{\prime \prime} \cdot \boldsymbol{n}\right|_{\Gamma_{2}}=0 .
$$

Здесь q" и $\mathbf{e}$ - симметричные тензоры, второго ранга напряжений и деформаций.

Согласно принципу суперпозиции напряженное состояние в теле задано теперь суммой тензоров $\varepsilon(x)+\mathbf{q}^{\prime \prime}(x), x \in V$. Будем считать, что тензор $\mathbf{q}^{\prime \prime}(x)$ оптимизирует напряженное состояние в упругом теле, если в некоторой наперед заданной области $V_{1} \subset V$ имеет место равенство

$$
\chi(x) \mathbf{q}^{\prime \prime}(x)+\chi(x) \boldsymbol{\sigma}(x)=0, \chi(x)=\left\{1, x \in V_{1} ; 0, x \notin V_{1}\right\} .
$$

Тогда задача оптимизации формулируется следующим образом: требуется найти такое поле первоначальных деформаций, при реализации которого возникнут такие дополнительные напряжения, что в области $V_{1}$ будет выполняться равенство (3).

\section{3. Функциональные пространства тензоров второго ранга}

В основу дальнейших рассуждений положим энергетическое гильбертово пространство $T$, состоящее из всевозможных симметричных тензоров напряжений, определенных в $V$ [19]. Скалярное произведение и норма в нем заданы формулами $[19,20]$

$$
\left(\boldsymbol{p}_{1}, \boldsymbol{p}_{2}\right)=\int_{V} \boldsymbol{p}_{\mathbf{1}} \cdot \boldsymbol{S} \cdot \boldsymbol{p}_{\mathbf{2}} d V ; \quad\|\boldsymbol{p}\|^{2}=(\boldsymbol{p}, \boldsymbol{p}) ; \boldsymbol{p}, \boldsymbol{p}_{1}, \boldsymbol{p}_{\mathbf{2}} \in T,
$$

где $\boldsymbol{S}$ - тензор модулей податливости $\boldsymbol{S}=\boldsymbol{C}^{-1}$. Известно $[19,20]$, что пространство $\boldsymbol{T}$ является ортогональной суммой следующих подпространств:

$$
T_{1}=\left\{\boldsymbol{p}^{\prime}: \boldsymbol{e}^{\prime}=\boldsymbol{S} \cdot \boldsymbol{p}^{\prime} ; \quad \text { Jnk } \boldsymbol{e}^{\prime}=0\right\} ; \quad T_{2}=\left\{\boldsymbol{p}^{\prime}: \nabla \cdot \boldsymbol{p}^{\prime}=0,\left.\boldsymbol{p}^{\prime \prime} \cdot \boldsymbol{n}\right|_{\Gamma}=0\right\}
$$

Оператор Jnk - опреатор несовместности [17].

Элементами подпространства $T_{1}$ являются решения краевой задачи теории упругости при всевозможных объемных и поверхностных силах: 


$$
\nabla \cdot \boldsymbol{p}^{\prime}=\boldsymbol{f} ; \boldsymbol{e}^{\prime}=\operatorname{def} \boldsymbol{u} ; \boldsymbol{p}^{\prime}=\boldsymbol{C} \cdot \boldsymbol{e}^{\prime} ;\left.\boldsymbol{n} \cdot \boldsymbol{p}^{\prime}\right|_{\Gamma}=\boldsymbol{t} .
$$

Здесь $\boldsymbol{f}=\boldsymbol{f}(x) ; x \in V$ - вектор объемных сил; $\boldsymbol{t}=\boldsymbol{t}(x) ; x \in \Gamma$ - вектор поверхностных сил. Элементами подпространства $T_{2}$ являются решения краевой задачи:

$$
\nabla \cdot \boldsymbol{p}^{\prime \prime}=0, \quad \boldsymbol{e}^{\prime}=\operatorname{def} \boldsymbol{u}, \quad \boldsymbol{p}^{\prime \prime}=\boldsymbol{C} \cdot\left(\boldsymbol{e}^{\prime}-\boldsymbol{\varepsilon}^{*}\right),\left.\quad \boldsymbol{n} \cdot \boldsymbol{p}^{\prime \prime}\right|_{\Gamma}=0 .
$$

для всевозможных симметричных тензоров второго ранга первоначальных деформаций $\boldsymbol{\varepsilon}^{*}$. Если $p^{\prime} \in T_{2}$, а $p^{\prime \prime} \in T_{2}$, то, используя формулу Остроградского-Гауса, находим:

$$
\begin{gathered}
\left(\boldsymbol{p}^{\prime \prime}, \boldsymbol{p}^{\prime}\right)=\int_{V} \boldsymbol{p}^{\prime \prime} \cdot \boldsymbol{S} \cdot \boldsymbol{p}^{\prime} d V=\int_{V} p^{\prime \prime} \cdot \cdot e^{\prime} d V=\int_{V} \boldsymbol{p}^{\prime \prime} \cdot \cdot \operatorname{def} \boldsymbol{u} d V= \\
=-\int_{V} \nabla \cdot \boldsymbol{p}^{\prime \prime} \cdot \boldsymbol{u} d V+\int_{\Gamma} \boldsymbol{n} \cdot \boldsymbol{p}^{\prime \prime} \cdot \boldsymbol{u} d \Gamma=0 .
\end{gathered}
$$

Рассмотрим еще два подпространства:

$$
D_{1}=\left\{\boldsymbol{q}^{\prime}: \operatorname{Jnk}\left(\boldsymbol{S} \cdot \boldsymbol{q}^{\prime}\right)=0 ;\left.\quad u\right|_{\Gamma_{1}}=0\right\} ; \quad D_{2}=\left\{\boldsymbol{q}^{\prime^{\prime}}: \nabla \cdot \boldsymbol{q}^{\prime}=0,\left.\boldsymbol{n} \cdot \boldsymbol{q}^{\prime \prime}\right|_{\Gamma_{2}}=0\right\} .
$$

Элементами подпространства $D_{1}$ являются решения краевой задачи

$$
\nabla \cdot \boldsymbol{q}^{\prime}=\boldsymbol{f} ; \quad \boldsymbol{e}^{\prime}=\operatorname{def} \boldsymbol{u} ; \quad \boldsymbol{q}^{\prime}=\boldsymbol{C} \cdot \boldsymbol{e}^{\prime} ;\left.\quad \boldsymbol{u}\right|_{\Gamma_{1}}=0 ;\left.\quad \boldsymbol{q}^{\prime} \cdot \boldsymbol{n}\right|_{\Gamma_{2}}=\boldsymbol{t}
$$

для всевозможных $\boldsymbol{f}$ и $\boldsymbol{t}$.

Элементы подпространства $D_{2}$ - решения краевой задачи

$$
\nabla \cdot \boldsymbol{q}^{\prime \prime}=\mathbf{0} ; \quad \boldsymbol{e}^{\prime}=\operatorname{def} \boldsymbol{u} ; \quad \boldsymbol{q}^{\prime \prime}=\boldsymbol{C} \cdot\left(\boldsymbol{e}^{\prime}-\boldsymbol{\varepsilon}^{*}\right) ;\left.\quad \boldsymbol{u}\right|_{\Gamma_{1}}=\boldsymbol{v} ;\left.\quad \boldsymbol{q}^{\prime \prime} \cdot \boldsymbol{n}\right|_{\Gamma_{2}}=0
$$

для различных $\boldsymbol{\varepsilon}^{*}$ и $V$.

Используя формулу Остроградского-Гаусса, находим:

$$
\begin{aligned}
& \left(\boldsymbol{q}^{\prime \prime}, \boldsymbol{q}^{\prime}\right)=\int_{V} \boldsymbol{q}^{\prime \prime} \cdot \cdot \boldsymbol{q}^{\prime} d V=\int_{V} \boldsymbol{q}^{\prime \prime} \cdot \cdot \boldsymbol{e}^{\prime} d V=\int_{V} \boldsymbol{q}^{\prime \prime} \cdot \cdot \operatorname{def} \boldsymbol{u} d V= \\
= & -\int_{V} \nabla \cdot \boldsymbol{q}^{\prime \prime} \cdot \boldsymbol{u} d V+\int_{\Gamma} \boldsymbol{n} \cdot \boldsymbol{q}^{\prime \prime} \cdot \boldsymbol{u} d \Gamma= \\
= & -\int_{V} \nabla \cdot \boldsymbol{q}^{\prime \prime} \cdot \boldsymbol{u} d V+\int_{\Gamma_{1}} \boldsymbol{n} \cdot \boldsymbol{q}^{\prime \prime} \cdot \boldsymbol{u} d \Gamma+\int_{\Gamma_{2}} \boldsymbol{n} \cdot \boldsymbol{q}^{\prime \prime} \cdot \boldsymbol{u} d \Gamma=0 .
\end{aligned}
$$

Таким образом, пространство $T$ есть ортогональная сумма либо подпространств $T_{1}$ и $T_{2}$, либо подпространств $D_{1}$ и $D_{2} ; T=T_{1} \oplus T_{2} ; D=D_{1} \oplus D_{2}$. 
Итак, любой тензор $\boldsymbol{p} \in T[19,20]$ можно представить единственным образом: суммой $\boldsymbol{p}=\boldsymbol{p}^{\prime}+\boldsymbol{p}^{\prime \prime}$ или суммой $\boldsymbol{p}=\boldsymbol{q}^{\prime}+\boldsymbol{q}^{\prime \prime}$, где $\boldsymbol{p}^{\prime} \in T_{1} ; \boldsymbol{p}^{\prime \prime} \in T_{2} ; \boldsymbol{p}^{\prime}, \boldsymbol{p}^{\prime \prime}=0 ; \boldsymbol{q}^{\prime} \in D_{1} ; \boldsymbol{q}^{\prime \prime} \in D_{2} ;$ $\boldsymbol{q}^{\prime}, \boldsymbol{q}^{\prime \prime}=0 ; \boldsymbol{p}^{\prime}=P_{1} \boldsymbol{p} ; \boldsymbol{p}^{\prime \prime}=P_{2} \boldsymbol{p} ; \boldsymbol{q}^{\prime}=Q_{1} \boldsymbol{p} ; \boldsymbol{q}^{\prime \prime}=Q_{2} \boldsymbol{p}$.

Здесь $P_{1}$ и $P_{2}$ - операторы ортогонального проектирования на подпространства $T_{1}$ и $T_{2}$ соответственно, а $Q_{1}$ и $Q_{2}$ - ортопроекторы на $D_{1}$ и $D_{2}$, причем $P_{1}+P_{2}=I ; Q_{1}+Q_{2}=I$, где $I$ - тождественный оператор. Далее очевидно, что $T_{2} \subset D_{2}$ и $D_{1} \subset T_{1}$. Тогда $Q_{1} P_{2}=$ $P_{2} Q_{1}=0[21,22]$ (проекторы $Q_{1}$ и $P_{2}$ ортогональны, так как ортогональны подпространства $D_{1}$ и $\left.T_{2}\right)$. И кроме того имеют место равенства:

$$
P_{1} Q_{1}=Q_{1} ; \quad Q_{1} P_{1}=Q_{1} ; Q_{2} P_{2}=Q_{2} ; P_{2} Q_{2}=Q_{2}
$$

Эти равенства проверяются непосредственно. Например:

$$
P_{1} Q_{1}=\left(I-P_{2}\right) Q_{1}=Q_{1}-P_{2} Q_{1}=Q_{1}
$$

и т. д. Отметим, наконец, что решения задач (1) и (2) принадлежит подпространству $D_{2}$.

\section{4. Решение системы (2)}

1. Найдем теперь общий вид решения системы (2). Задачу будем решать в два этапа. Сначала запишем решение задачи (5). В работе [18] показано, что тензор напряжений здесь определяется выражением $\boldsymbol{p}^{\prime \prime}=-P_{2} \boldsymbol{\sigma}^{*}\left(\boldsymbol{\sigma}^{*}=C \cdot \cdot \varepsilon^{*}\right)$, а совместные деформации и перемещения являются решениями уравнений (4) при $f=\nabla \cdot \boldsymbol{\sigma}^{*}, \mathrm{t}=\left.\boldsymbol{\sigma}^{*} \cdot n\right|_{\Gamma}$ (задача (А)), причем $\boldsymbol{p}^{\prime}=P_{1} \boldsymbol{\sigma}^{*}$. Итак, получили напряженно-деформированное состояние в теле со свободной границей при заданном поле первоначальных деформаций. Обозначим через $\boldsymbol{w}$ перемещения, которые получают при этом точки границы $\left(\left.\boldsymbol{u}\right|_{\Gamma}=\boldsymbol{w}\right)$. На втором этапе необходимо решить задачу (1) при граничных условиях $\left.\boldsymbol{u}\right|_{\Gamma_{1}}=-w,\left.\boldsymbol{\sigma} \cdot \boldsymbol{n}\right|_{\Gamma_{2}}=0$ (задача (В)) и затем наложить это решение на решение задачи (5).

Рассмотрим дополнительную систему

$$
\nabla \cdot \boldsymbol{p}_{w}^{\prime}=\nabla \cdot \boldsymbol{\sigma}^{*}, \boldsymbol{e}^{\prime}=\operatorname{def} \boldsymbol{u}, \boldsymbol{p}_{w}^{\prime}=\boldsymbol{C} \cdot \boldsymbol{e}^{\prime},\left.\boldsymbol{u}\right|_{\Gamma_{1}}=w,\left.\boldsymbol{p}_{w}^{\prime} \cdot \boldsymbol{n}\right|_{\Gamma_{2}}=\left.\boldsymbol{\sigma}^{*} \cdot \boldsymbol{n}\right|_{\Gamma_{2}}
$$

Решение системы (7), очевидно, совпадает с решением задачи (А). Следовательно, $\boldsymbol{p}^{\prime}{ }_{w}=\boldsymbol{p}^{\prime}=P_{1} \boldsymbol{\sigma}^{*}$. Из данного тензора $\boldsymbol{p}^{\prime}{ }_{w}$ вычтем тензор напряжений $\boldsymbol{\sigma}_{w}$, удовлетворяющий системе (1) с граничными условиями $\left.\boldsymbol{u}\right|_{\Gamma_{1}}=w,\left.\boldsymbol{\sigma}_{w} \cdot \boldsymbol{n}\right|_{\Gamma_{2}}=0$. Тогда $\boldsymbol{p}_{w}^{\prime}-\boldsymbol{\sigma}_{w} \in D_{1}$. Отсюда $\boldsymbol{p}_{w}^{\prime}=\left(\boldsymbol{p}_{w}^{\prime}-\boldsymbol{\sigma}_{w}\right)+\boldsymbol{\sigma}_{w}$, где $\boldsymbol{\sigma}_{w} \in D_{2}$.

То есть имеет место разложение тензора $\boldsymbol{p}^{\prime}{ }_{w}$ на сумму элементов из подпространства $D_{1}$ и $D_{2}$. В силу единственности такого представления находим, что $\boldsymbol{\sigma}_{w}=Q_{2} \boldsymbol{p}_{w}^{\prime}=Q_{2} P_{1} \boldsymbol{\sigma}^{*}$. Теперь решение задачи (В) определяется выражением $\left(-Q_{2} P_{1} \boldsymbol{\sigma}^{*}\right)$. Итак, искомое решение задачи (2) с учетом равенства (6) можно представить в виде:

$$
\begin{gathered}
\boldsymbol{q}^{\prime \prime}=-P_{2} \boldsymbol{\sigma}^{*}-Q_{2} P_{1} \boldsymbol{\sigma}^{*}=-\left(I-P_{1}\right) \boldsymbol{\sigma}^{*}-Q_{2} P_{1} \boldsymbol{\sigma}^{*}=-\boldsymbol{\sigma}^{*}+\left(I-Q_{2}\right) P_{1} \boldsymbol{\sigma}^{*}=-\boldsymbol{\sigma}^{*}+ \\
+Q_{1} P_{1} \boldsymbol{\sigma}^{*}=-\boldsymbol{\sigma}^{*}+Q_{1} \boldsymbol{\sigma}^{*}=-\left(I-Q_{1}\right) \boldsymbol{\sigma}^{*}=-Q_{2} \boldsymbol{\sigma}^{*}
\end{gathered}
$$

\section{5. Метод решения задачи оптимизации}

Следуя работе [20], перепишем уравнение (3) с учетом равенства (8) в виде $\chi Q_{2} \boldsymbol{\sigma}^{*}=\chi \boldsymbol{\sigma}$. Подставляя сюда выражение $Q_{2}=I-Q_{1}$ получаем операторное уравнение $\chi \boldsymbol{\sigma}^{*}=\chi Q_{1} \boldsymbol{\sigma}^{*}+\chi \boldsymbol{\sigma}$. 
Будем искать решение данного уравнения на множестве $\chi T$. Если $\boldsymbol{\sigma}^{*} \in \chi T$, то $\chi \boldsymbol{\sigma}^{*}=$ $\boldsymbol{\sigma}^{*},(\chi \chi=\chi)$. Тогда окончательно

$$
\boldsymbol{\sigma}^{*}=\chi Q_{1} \boldsymbol{\sigma}^{*}+\chi \boldsymbol{\sigma}
$$

Оценим норму оператора $\chi Q_{1}$. Имеем ||$\chi Q_{1}\|\leq\| \chi\left\|\cdot|| Q_{1}\right\|$. Известно, что ||$Q_{1} \|=1$ [14]. Далее $\|\chi p\|^{2}=\int_{V} \chi p \cdots S \cdots \chi p d \boldsymbol{v}=\int_{V_{1}} p \cdots p d V<\int_{V} p \cdot S \cdots p d V=\|p\|^{2}$. Здесь $p \cdots S \cdots p$ - положительно определенная квадратичная форма, $p$ - некоторый элемент из множества $M \subset T$, которое составляют тензоры, определенные в области $V^{\prime}$, где $V^{\prime}-$ любая область? входящая или совпадающая с $V$, причем $V_{1} \subset V^{\prime}$. Тогда, рассматривая $\chi$ как оператор, действующий из $M \subset T$ в $\chi T$, получаем ||$\chi||<1$. Если же $\chi$ действует из $\chi T$ в $\chi T$, то ||$\chi||=1$. Очевидно, что оператор $Q_{1}$ отображает элементы из $T$ в $M$, кроме элементов $\chi q^{\prime} \in D_{1}$. Отсюда следует, что оператор $\chi Q_{1}$, определенный на множестве $\chi T\left(\chi T \cap D_{1}\right)$, имеет норму ||$\chi Q_{1}||<1$. Следовательно, он является оператором сжатия, и решение уравнения (9) представимо сходящимся рядом

$$
\boldsymbol{\sigma}^{*}=\sum_{n=0}^{\infty}\left(\chi Q_{1}\right)^{n} \chi \boldsymbol{\sigma} .
$$

Наконец, искомое поле первоначальных деформаций определяется тензором $\boldsymbol{\varepsilon}^{*}=\boldsymbol{S} \cdot \boldsymbol{\sigma}^{*}$.

Замечание 1. Так как $Q_{2}\left(\boldsymbol{\sigma}^{*}+\boldsymbol{q}^{\prime}\right)=Q_{2} \boldsymbol{\sigma}^{*}$, где $\boldsymbol{q}^{\prime}-$ произвольный элемент из $D_{1}$, то любой тензор $\boldsymbol{\varepsilon}=\boldsymbol{\varepsilon}^{*}+\boldsymbol{\varepsilon}^{\prime}$, где $\boldsymbol{\varepsilon}^{\prime}=\boldsymbol{S} \cdot \boldsymbol{q}^{\prime}$, также инициирует поле напряжений $\boldsymbol{q}^{\prime}(x)$, обладающее свойством (3).

Замечание 2. Если в теле требуется создать заданное поле напряжений $\boldsymbol{q}^{\prime}(x)$, то поле первоначальных деформаций определяется тензором $\boldsymbol{\varepsilon}^{*}(x)=\boldsymbol{\varepsilon}^{\prime \prime}(x)+\boldsymbol{\varepsilon}^{\prime}(x)$, где $\boldsymbol{\varepsilon}^{\prime \prime}=\boldsymbol{S} \cdot \boldsymbol{q}^{\prime \prime}$; $\boldsymbol{\varepsilon}^{\prime}-$ произвольный тензор совместных деформаций, которому отвечают перемещения, обращающиеся в нуль на границе $\Gamma_{1}$.

\section{6. Пример}

Рассмотрим частный случай. Пусть точкам границы тонкого круглого диска радиуса $R$ заданы радиальные перемещения $v$. Требуется найти первоначальные деформации, вызывающие появление таких добавочных напряжений, которые в сумме с напряжениями от внешней нагрузки дают нулевое напряженное состояние в центральной зоне радиуса $R_{1}$.

Решения краевых задач (1) и (2) определяют соответственно формулы:

$$
\begin{gathered}
\sigma_{r}=\sigma_{\theta}=\frac{E v}{(1-\mu) R^{\prime}}, \quad \varepsilon_{r}=\varepsilon_{\theta}=\frac{v}{R} ; \\
q_{r}^{\prime \prime}=q_{\theta}^{\prime \prime}=\frac{E \varepsilon_{0}^{*}}{2(1-\mu)}\left[(1+\mu) \frac{R_{1}^{2}}{R^{2}}+(1-\mu)\right], \\
e_{r}=e_{\theta}=\frac{1}{2} \varepsilon_{0}^{*}(1+\mu)\left(1-\frac{R_{1}^{2}}{R^{2}}\right), \quad 0 \leq r \leq R_{1}, \\
q_{r, \theta}^{\prime \prime}=\frac{E \varepsilon_{0}^{*} R_{1}^{2}}{2 R^{2}(1-\mu)}\left[(1+\mu) \pm(1-\mu) \frac{R^{2}}{r^{2}}\right], \\
e_{r, \theta}=-\frac{\varepsilon_{0}^{*}(1+\mu) R_{1}^{2}}{2 R^{2}}\left(1 \pm \frac{R_{1}^{2}}{R^{2}}\right), \quad R_{1} \leq r \leq R .
\end{gathered}
$$


Индексами $r$ и $\theta$ обозначены, соответственно, радиальные и тангенциальные напряжения и деформации; $E$ - модуль Юнга; $\mu$ - коэффициент Пуассона; $\varepsilon_{0}^{*}=\varepsilon_{r}^{*}=\varepsilon_{\theta}^{*}-$ первоначальные деформации в центральной зоне, требующие определения.

Используя данные выражения, находим, что в формуле (10) величина

$$
\chi \sigma=\chi \frac{E v}{(1-\mu) R}
$$

и оператор

$$
Q_{1}=\frac{1}{2}(1+\mu)\left(1-\frac{R_{1}^{2}}{R^{2}}\right),
$$

где $\chi=1, r \in\left[0, R_{1}\right] ; \chi=0, r \in\left[R_{1}, R\right]$. Тогда суммируя ряд (10), который представляет собой убывающую геометрическую прогрессию, получаем:

$$
\sigma_{0}^{*}=\chi \sigma_{r}^{*}=\chi \sigma_{\theta}^{*}=\frac{2 \chi R v E}{(1-\mu)\left[(1-\mu) R^{2}+(1+\mu) R_{1}^{2}\right]} .
$$

Отсюда искомое значение $\varepsilon_{0}^{*}=(1-\mu) E^{-1} \sigma_{0}^{*}$. Далее получаем:

$$
\begin{gathered}
q_{r}^{\prime \prime}=q_{\theta}^{\prime \prime}=\frac{E \mu}{(1-\mu) R} ; 0 \leq r \leq R_{1} ; \\
q_{r, \theta}^{\prime \prime}=\frac{E R^{2} v}{R(1-\mu)\left[(1-\mu) R^{2}+(1+\mu) R_{1}^{2}\right]}\left[(1+\mu) \pm(1-\mu) \frac{R^{2}}{r^{2}}\right] ; \quad R_{1} \leq r \leq R .
\end{gathered}
$$

Таким образом, суммарные напряжения в центральной зоне равны нулю, а в кольце, окружающем эту область, получаем решение задачи Ляме для соответствующих граничных условий.

\section{Литература}

1. Биргер И. А. Остаточные напряжения. - М. : Машгиз, 1963. - 262 с.

2. Поздеев А. А., Няшин Ю. И., Трусов П. В. Остаточные напряжения: теория и приложения. - М. : Наука, 1982. - 111 с.

3. Павлов В. Ф., Кирпичев В. А., Иванов В. Б. Остаточные напряжения и сопротивление усталости упрочненных деталей с концентраторами напряжений. - Самара : Самар. науч. центр РАН, 2008. - 64 с.

4. Абрамов В. В. Остаточные напряжения и деформации в металлах. - М. : Машгиз, 1961. -355 c.

5. Технологические причины возникновения остаточных напряжений / А. В. Королев, А. А. Мазина, А. С. Яковишин, А. В. Шалунов // Современные материалы, техника и технологии. - 2016. - № 5 (8). - С. 116-119.

6. Struzhanov V. V. Determination of shrinkage stresses in components of stochastically reinforced composites // Soviet Applied Mechanics. - 1982. - Vol. 18, no. 5. - P. 445-449. DOI: $10.1007 / \mathrm{bf00883786.}$

7. Шинкин В. Н. Остаточные напряжения при экструдировании стальной трубы // Молодой ученый. - 2015. - № 20 (100). - С. 88-95. 
8. К Колмогоров Г. Л., Кузнецова Е. В., Тиунов В. В. Технологические остаточные напряжения и их влияние на долговечность и надежность металлоизделий. - Пермь : Изд-во Перм. нац. исслед. политехнического ун-та, 2012. - 226 с.

9. Буркин С. П., Шимов Г. В., Андрюкова Е. А. Остаточные напряжения в металлопродукции : учебное пособие. - Екатеринбург : Изд-во Уральского ун-та, 2015. - 248 с.

10. Hosford W. F. Mechanical Behavior of Materials. - Cambridge : Cambridge University Press, 2005. $-421 \mathrm{p}$.

11. Grack arrest in thin metallic film stacks due to material and residual stress inhomogeneities / D. Kozic, H.-P. Gдnser, R. Brunner, D. Kienerb, T. Antretterc, O. Kolednik // Thin solid Films. 2018. - Vol. 668. - P. 14-22. - DOI: 10.1016/j.tsf.2018.10.014.

12. Коновалов Д. А., Вичужанин Д. И., Смирнов С. В. Оценка остаточных напряжений методом внедрения индентора // Труды Второй Всероссийской научной конференции «Математическое моделирование и краевые задачи», 1-3 июня 2005 г. - СамГТУ, Самара, 2005. - Ч. 1 : Математические модели механики, прочность и надежность конструкций. C. $155-157$.

13. Фишкин А. И. Применение метода регуляризации Тихонова для определения остаточных напряжений // Строительная механика инженерных конструкций и сооружений. 2009. - № 4. - С. 48-55.

14. Кудрявцев И. В. Внутренние напряжение как резерв прочности в машиностроении. М. : Машгиз, 1951. - 278 с.

15. Рожков И. И., Мыльников В. В. Расчет внутренних остаточных напряжений, возникающих в закаленных деталях после химико-термической обработки // Международный журнал экспериментального образования. - 2014. - № 1. - С. 114-118.

16. Синчурин Д. В. Влияние метода дроструйного упрочнения на повышение эксплуатационной надежности деталей // Молодой ученый. - 2015. - № 21.2 (101.2). - С. 54-57.

17. Лурье А. И. Теория упругости. - М. : Наука, 1970. - 940 с.

18. Sokolov A. G., Struzhanov V. V. A problem of optimizing the stressed state in an elastic solid // Journal of Applied Mathematics and Mechanics. - 2001. - Vol. 65, iss. 2. - P. 311-316. DOI: 10.1016/S0021-8928(01)00035-1.

19. Михлин С. Г. Вариационные методы в математической физике. - М. : Наука, 1970. 512 c.

20. Стружанов В. В. О методе ортогональных проекций в теории упругости // Вестн. Сам. гос. техн. ун-та. Сер. Физ-мат. Науки. - 2017. - Т. 21, № 2. - С. 308-325. DOI: $10.14498 /$ vsgtu1542.

21. Канторович А. В., Акилов Г. П. Функциональный анализ. - М. : Наука., 1977. - 744 с.

22. К Константинов Р. В. Лекции по функциональному анализу. - М. : МФТИ, 2009. - 374 с. 